\title{
Imaging Cluster Surfaces with Atomic Resolution: The Strong Metal-Support Interaction State of Pt Supported on $\mathrm{TiO}_{2}(110)$
}

\author{
Olga Dulub, Wilhelm Hebenstreit, and Ulrike Diebold* \\ Department of Physics, Tulane University, New Orleans, Louisiana 70118
}

(Received 22 October 1999)

\begin{abstract}
Nanosized platinum clusters were grown on a $\mathrm{TiO}_{2}(110)$ surface and annealed in ultrahigh vacuum at high temperatures. This leads to the so-called strong metal-support interaction (SMSI) state, characterized by a complete encapsulation of the clusters with a reduced titanium oxide layer. We present atomically resolved scanning tunneling microscopy measurements of the cluster surfaces and an atomic model of the SMSI state. The ability to resolve the cluster surface geometry with atomistic detail may help to identify the active sites responsible for the SMSI.
\end{abstract}

PACS numbers: 68.35.Bs, 61.16.Ch, 73.40.Rw, 82.65.Jv

Nanosized clusters exhibit unique properties and play a role in many technical applications. Detailed structural information about their surfaces is critical to a deeper understanding of their physics and chemistry. Scanning tunneling microscopy (STM) allows atomic-scale investigation of the electronic and geometric structure of surfaces, but almost no atomically resolved STM results of nanosized clusters thicker than a few atomic layers are currently available [1], because the finite size of the STM tip leads to convolution effects which severely disturb measurements.

One specific example for the lack of structural knowledge of nanosized cluster surfaces is the so-called "strong-metal support interaction" (SMSI) in heterogeneous catalysis. SMSI has been termed by Tauster et al. [2] to account for the changes in catalytic activity when the group VIII metals $\mathrm{Fe}, \mathrm{Ni}$, Rh, Pt, Pd, and Ir, supported on reducible oxides $\left(\mathrm{TiO}_{2}, \mathrm{TaO}_{5}, \mathrm{CeO}_{2}, \mathrm{NbO}\right.$, etc.), are reduced at elevated temperature. Adsorption of $\mathrm{H}_{2}$ and $\mathrm{CO}$ is drastically reduced, but competitive hydrogenation versus hydrogenolysis reactions are greatly favored in SMSI systems. For example, methanation production from $\mathrm{CO}$ or $\mathrm{CO}_{2}$ and $\mathrm{H}_{2}$ is enhanced by 3 orders of magnitude [3]. Because SMSI allows one to tailor the selectivity of a catalyst, it has caused widespread interest.

High-resolution electron microscopy (HREM) clearly shows that some sort of reduced titanium oxide migrates onto the clusters [4]. The composition and geometric structure of the encapsulation layer needs to be known to develop a realistic picture of reaction mechanisms. The encapsulation layer is only a few atomic layers thick, making impossible selective diffraction in HREM. STM studies of the single-crystalline SMSI model systems $\mathrm{Rh} / \mathrm{TiO}_{2}(001)$ [5], $\mathrm{Rh} / \mathrm{TiO}_{2}$ (110) [6], $\mathrm{Pd} / \mathrm{TiO}_{2}(110)$ [7], and $\mathrm{Ir} / \mathrm{TiO}_{2}(110)$ [8] have yielded information about the general morphology and size distribution of clusters only. The SMSI state was investigated using "reverse model catalysts" where oxide islands were grown on flat single crystals [9]. It is arguable whether such structures resemble the self-organized layers that evolve on clusters upon high-temperature annealing. In fact, as we will show for the case of $\mathrm{Pt}$, the encapsulation layer does not resemble the structure of $\mathrm{TiO}_{x}$ films on $\mathrm{Pt}(111)$ [10].

We have studied $\mathrm{Pt}$ on $\mathrm{TiO}_{2}(110)$ in the SMSI state. Low-energy $\mathrm{He}^{+}$ion scattering (LEIS) and scanning tunneling spectroscopy (STS) results confirm the encapsulation of Pt clusters after annealing in UHV at high temperatures. Most clusters exhibit a regular hexagonal shape with flat tops and an average size of $200 \AA$. Atomicresolution STM reveals the surprising geometric structure of the encapsulation layer, i.e., a hexagonal striped domain structure with apparent in-plane $A B C$ stacking. We propose an atomic model, consisting of two layers of titanium oxide, to rationalize the structure. The layer is polar, and its thickness is self-limited. These results are important because they (i) represent the first case of formation of a self-organized, polar oxide layer; (ii) might represent an important step in understanding the nature of strong metal-support interactions; and (iii) the special shape of the nanosized clusters allows for one of the first STM studies with atomic resolution of cluster surfaces.

The experiments were performed in ultrahigh vacuum (UHV). A bulk-reduced $\mathrm{TiO}_{2}(110)$ single crystal sample was cleaned by repeated cycles of sputtering and annealing. Pt was evaporated onto the $\mathrm{TiO}_{2}(110)$ surface at room temperature. The average layer thickness is determined using a quartz crystal monitor. Pt forms three-dimensional clusters at room temperature [11]. After deposition, the sample was heated to $200{ }^{\circ} \mathrm{C}$ for $30 \mathrm{~min}$ (to increase cluster size); to $700{ }^{\circ} \mathrm{C}$ for $5 \mathrm{~min}$, and to $500{ }^{\circ} \mathrm{C}$ for $30 \mathrm{~min}$. STM was performed at room temperature in the constant current topographic (CCT) mode. Shown are empty states STM images taken with a positive sample bias voltages ranging between 1.2 and $2.5 \mathrm{~V}$. Filled-states CCT's with negative sample bias voltage gave similar results but fuzzier images. The STM was calibrated with atomic resolution images of clean $\mathrm{TiO}_{2}(110)(1 \times 1)$.

LEIS measures the composition of the topmost surface layer. Each atomic mass leads to a distinct peak; see Fig. 1a. After deposition of 25 monolayers (ML) of Pt at room temperature, LEIS shows an intense Pt peak while the 

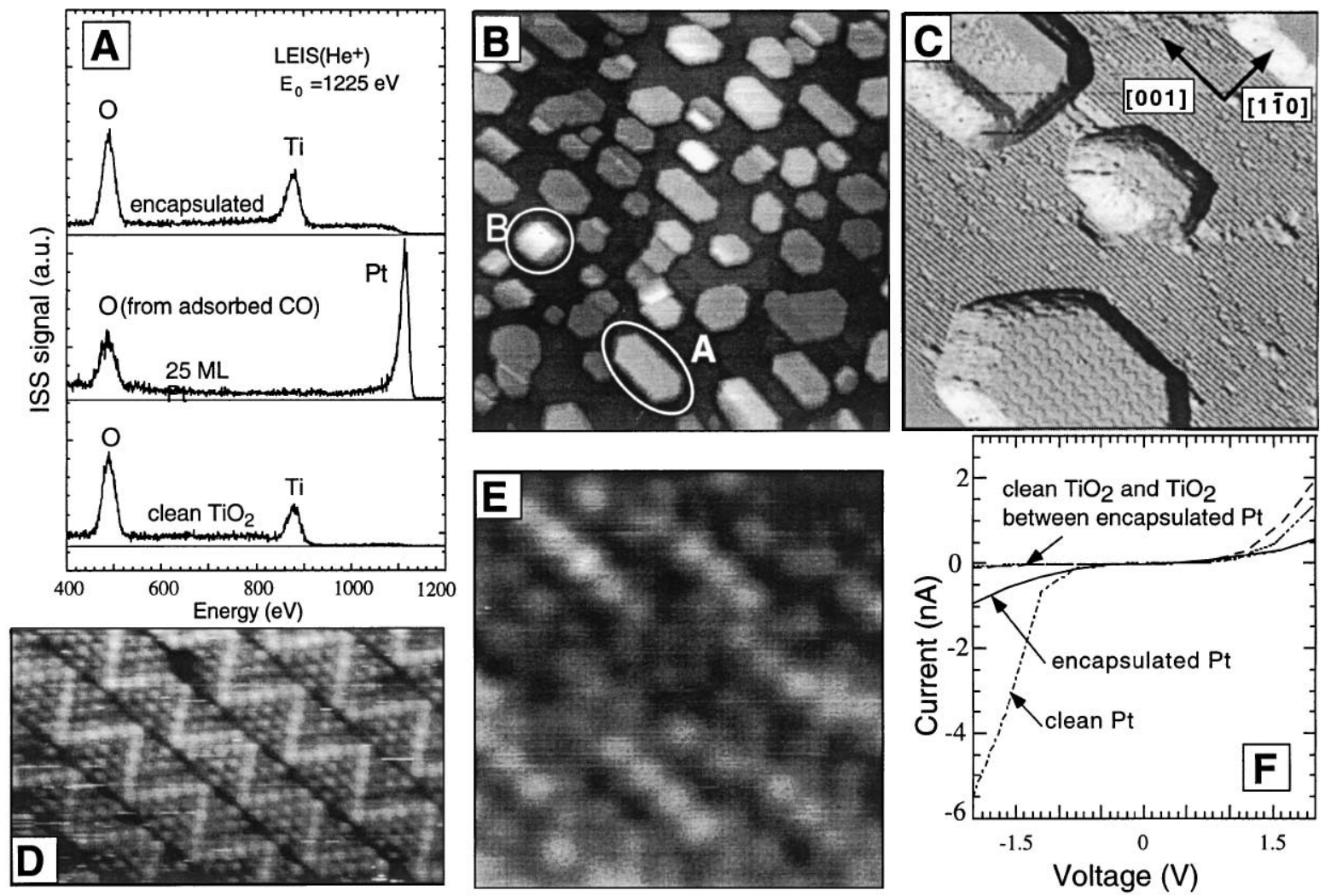

FIG. 1. (a) Low-energy $\mathrm{He}^{+}$ion scattering (LEIS) spectra of (bottom) the clean $\mathrm{TiO}_{2}(110)$ surface, (center) after evaporation of 25 monolayers $\mathrm{Pt}$ at room temperature, and (top) after the high-temperature treatment causing encapsulation. (b)-(e) STM results after the high-temperature treatment. (b) Overview $(2000 \AA \times 2000 \AA)$. Clusters are approximately $200 \AA$ wide and $40 \AA$ high. Most clusters show hexagonal shape elongated along the substrate [001] direction (type $A$ ). A few square clusters (type $B$ ) are seen. (c) Small-scale image (500 $\mathrm{A} \times 500 \AA$ ), filtered to show the structure of the encapsulation layer on type- $A$ clusters. (d) Atomic-resolution image of an encapsulated "type- $A$ " cluster. (e) Atomic-resolution image of a square "type- $B$ " cluster, showing an amorphous overlayer. (f) STS of the different surfaces.

Ti peak disappears; the surface is completely covered with Pt. (The oxygen peak in Fig. 1a, center, is attributed to adsorption of $\mathrm{CO}$ from the residual gas. Because $\mathrm{CO}$ desorbs when the sample is heated to $270{ }^{\circ} \mathrm{C}$, it should have no effect on the encapsulation process.) After the heating excursion described above, the Pt peak is no longer present in the LEIS spectrum. Pt clusters are covered completely with a titanium oxide layer. The increased background at energies $<1100 \mathrm{eV}$ in Fig. 1a (top) indicates Pt in the near-surface region. From LEIS O to Ti intensity ratios, and the area covered with encapsulated clusters obtained with STM, we estimate the O:Ti ratio on the cluster surfaces as 2:1.

Figures $1 \mathrm{~b}-1 \mathrm{e}$ display a series of STM images of the encapsulated Pt clusters. Most clusters (type $A$ ) have a hexagonal shape elongated along the substrate [001] direction and are, on average, $40 \AA$ high and $200 \AA$ wide. A few have a square shape (type $B$ ). Those are smaller. A simple calculation (taking into account the deposited amount of Pt (25 ML), the surface coverage after encapsulation $(\sim 40 \%)$, and the cluster height shows that the clusters resemble "icebergs" reaching several tenths of angstroms deep into the substrate. Recent model studies have shown that massive restructuring takes place on reduced $\mathrm{TiO}_{2}$ crystals at relatively low temperature. For ex- ample, $\mathrm{Pd}$ clusters promote the growth of additional $\mathrm{TiO}_{2}$ layers at their perimeter; this can even lead to a complete burial of the clusters [12].

The $500 \AA \times 500 \AA$ large image in Fig. 1c is filtered to visualize the $3 \mathrm{D}$ structure of the encapsulated clusters and the substrate. The tops of the clusters are smooth and flat, which is essential for atomically resolved STM measurements. (The Pt particles appear similar to the "pillbox" shape observed in HREM images [13].) On top of the type- $A$ clusters, striped "zigzags" are visible. On different clusters the stripes are oriented either parallel to the substrate [001] direction or rotated by $\pm 60^{\circ}$. No clear preference for any rotational orientation was observed, nor a strict correlation between the directions of stripes and cluster elongations. Figure $1 \mathrm{~d}$ is an atomically resolved image of a type- $A$ cluster surface. The stripes are approximately $15 \AA$ wide and consist of bright spots arranged in a hexagonal symmetry with a distance of $3 \AA$. The bright zigzag rows contain either 5 or 6 atoms along the close-packed directions and separate triangular areas consisting of ten atoms. Surfaces of type- $B$ square clusters exhibit no apparent long-range order (Fig. 1e) with strings containing three to six atoms oriented along the substrate [001] direction. The "type- $B$ " clusters are probably crystallites with their 
(100) face parallel to the substrate. It is conceivable that such surfaces should be found at the sides of the hexagonal, (111)-oriented "type- $A$ " clusters.

STM current vs voltage $(I-V)$ curves have been taken from the clean sputter-annealed $\mathrm{TiO}_{2}(110)$ surface, from $\mathrm{TiO}_{2}(110)$ between encapsulated clusters, and from clusters of clean and encapsulated Pt (Fig. 1f). There is almost no difference in the $I-V$ curves from clean $\mathrm{TiO}_{2}$ and from $\mathrm{TiO}_{2}$ between encapsulated clusters. The electronic structure of the substrate is not strongly affected by the encapsulated clusters. The electronic structure of the clusters changes from those typically observed for small metal clusters on $\mathrm{TiO}_{2}$ [7] ("clean Pt") to a more semiconductorlike behavior after encapsulation.

Encapsulated Pt clusters on $\mathrm{TiO}_{2}$ substrates are very inert to adsorption from the residual gas in the UHV chamber. STM measurements of the same surface were possible for several days without any noticeable contamination. This loss of surfaces' ability to chemisorb $\mathrm{CO}$ and $\mathrm{H}_{2}$ following high temperature treatment is caused by the physical blockage of Pt adsorption sites and is characteristic for the SMSI effect [2].

An STM image taken on top of an encapsulated type- $A$ cluster is shown in Fig. 2. The local maxima of the bright spots are marked with a small dot. Neighboring stripe domains with zigzags are slightly shifted with respect to each other. The two parallel lines in Fig. 2a indicate that the rows of atoms in each stripe are in line with those of the third following stripe, creating an apparent in-plane stacking of $A B C A B C \ldots$ type. (The image has been carefully compensated for distortions caused by thermal drifts and piezo-non-linearities to allow this conclusion.) The stacking is also apparent from the relative positions of atoms next to the dark lines between the strip domains; they are displaced from each other by a horizontal vector of length $2 a \sqrt{3} / 3$ (with $a$ the length of the hexagonal unit cell). An $A B C$-type stacking is very unusual for hexagonal layers. It can be explained by a uniaxial expansion of the stripe domains, as will be shown below.

The atomic model of the encapsulation layer is displayed in Fig. 2. It consists of two layers of titanium oxide (side view Fig. 2). Previous studies of the encapsulation layer revealed its small thickness. When the sample was exposed to a continuous flux of $\mathrm{Ne}^{+}$ions, Pt immediately reappeared on the surface [11]. A HREM study confirmed that the encapsulation is only a few layers thick [14]. We assume [11] that a $\operatorname{Pt}(111)$ face is the "substrate" for the encapsulation layer (small filled circles in Fig. 2). This agrees with HREM studies of $\mathrm{Pt} / \mathrm{TiO}_{2}$ catalysts that showed crystalline overlayers on well-faceted $\{111\}$ surfaces, whereas other surfaces had disordered overlayers, similar to type- $B$ clusters described above [4]. XPS measurements do not show any evidence for Pt-Ti alloy formation that is expected at higher annealing temperatures [15].

The upper left corner of the model in Fig. 2 displays the top layer of the encapsulating titanium oxide structure, and is to be compared with the STM images in Figs. 1d
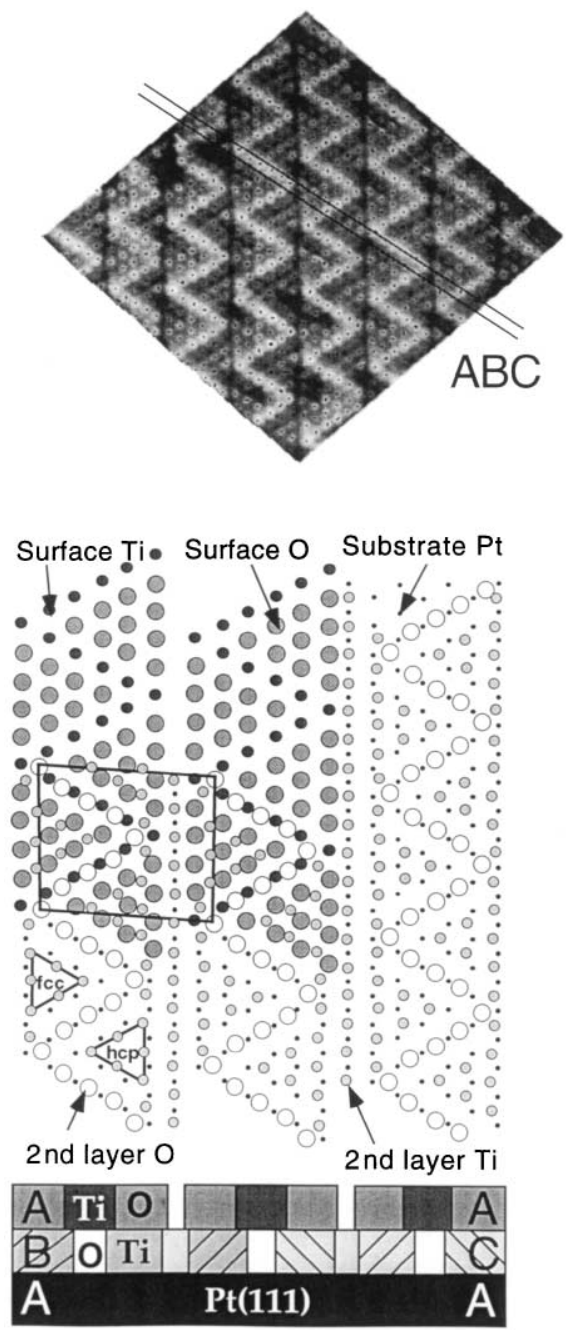

FIG. 2. (top) Atomically resolved STM image (100 $\left.\AA^{2}\right)$ of an encapsulated hexagonal cluster. The image is compensated for distortion. Atomic positions are indicated. (bottom) Top and side view of the proposed atomic model for the encapsulation layer on type- $A$ clusters; see text.

and 2. The chemical inertness of encapsulated clusters suggests either an oxygen-terminated surface or a saturation of bonds of surface $\mathrm{Ti}$ atoms. The contrast between different atoms in STM images could be due to higher positions of atoms in the zigzags, or they could be a different species, or both. All STM images are empty-state CCT's and the LDOS of unoccupied states is generally higher for titanium than for oxygen [16]. It is suggestive to associate bright spots on the image with $\mathrm{Ti}$, and darker spots with $\mathrm{O}$ atoms. Six Ti atoms separate triangles of ten oxygen atoms. The 20 oxygen and ten Ti atoms per unit cell are consistent with the LEIS O:Ti intensity ratio.

$\mathrm{Ti}$ atoms in the 2nd layer are placed in (approximately, see below) threefold hollow positions of the Pt fcc(111) substrate. Fcc and hcp stacked in domains contain eight titanium atoms each. (These are labeled $B$ and $C$ and marked by forward and back-slashed regions in the side view of Fig. 2.) They are separated by domain boundaries consisting of titanium and oxygen atoms in bridge positions. 
Five oxygen atoms form zigzags, which are covered by $\mathrm{Ti}$ atoms in the top layer. Rows of 2 nd layer titanium atoms separate the stripe domains and appear as the dark lines in STM. The second layer contains eight atoms of oxygen and 21 atoms of titanium per unit cell, amounting to an overall composition close to $\mathrm{TiO}_{1.1}$ in the two-layer film. This is consistent with previous measurements indicating a reduced titanium oxide [11]. The film exhibits a dipole moment, caused by the uneven distribution of negative and positive charges between the O-rich first and Ti-rich second layer, respectively. Polar films can be stabilized by the metallic substrate, but are limited to a thickness of a few atomic layers only. This is probably the physical reason for the self-limited thickness of the encapsulation layer.

Atoms in the top layer are placed in (approximately) on-top (or $A-A$ ) positions above platinum atoms of the substrate (see side view in Fig. 2). Ti atoms in zigzags reside in bridge positions above the second layer oxygen atoms, and are sixfold coordinated (counting four oxygen neighbors in-plane and two underneath). This high coordination number may cause the small reactivity towards gas adsorption, although the bonding geometry is far from the octahedral symmetry found in many oxide compounds. In a hard-ball model, surface Ti atoms are located $3.5 \AA$ above substrate Pt; elevated by $1 \AA$ above than the triangular domains of surface oxygens.

To accommodate the apparent $A B C A B C \ldots$ stacking observed in the top layer (Fig. 2, top), both Ti-O layers are expanded perpendicular to the stripes by a factor of $16 / 15$. This uniaxial expansion causes all the atoms to slightly shift from their perfect hollow or bridge positions. Domains of differently stacked atoms and partially incommensurate overlayers are common ways to accommodate strain relief caused by lattice-mismatched in ultrathin film metals on metal substrates. In this case, the lattice constants of a film with (almost) $\mathrm{TiO}$ stoichiometry is placed onto a $\mathrm{Pt}(111)$ substrate (containing $1.50 \times$ $10^{15}$ atoms $/ \mathrm{cm}^{2}$ per layer). The atomic density of the surface layer in the model, $1.29 \times 10^{15}$ atoms $/ \mathrm{cm}^{2}$, is reasonably close to the density of a $\mathrm{TiO}(111)$ layer $(1.32 \times$ $10^{15}$ atoms $/ \mathrm{cm}^{2}$ per layer). The observed lattice constant, $3 \AA$, compares well to interatomic distances on a $\mathrm{TiO}(111)$ surface $(2.95 \AA)$.

How relevant are these results to an understanding of the SMSI effect? While planar "model catalysts" have contributed much to a clarification of reaction models [9], there is always the caveat that single-crystalline materials in the UHV environment may exhibit very different structural properties as compared to catalysts exposed to high-temperature/high-pressure reaction conditions. The similarity between the clusters observed in this work with structural investigations of real catalysts $[4,13]$ gives us confidence that these two systems are structurally comparable. There is now general agreement that the enhanced catalytic activity in $\mathrm{Pt} / \mathrm{TiO}_{2}$ catalysts is a local effect. Special $\mathrm{Ti}^{3+}$ sites at the modified Pt catalyst, or Pt sites next to the reduced $\mathrm{TiO}_{x}$ layer are thought to be responsible for the dramatic enhancement in hydrogenation activity [3]. Possibly, the zigzag Ti atoms with the very peculiar, asymmetric coordination to neighboring oxygen atoms may play a role.

Summarizing, the first atomically resolved STM images on nanoscale clusters in the SMSI state show a wellordered overlayer on (111)-oriented Pt clusters supported on a $\mathrm{TiO}_{2}(110)$ substrate, as well as amorphous overlayers on square clusters. The proposed atomic model for the crystalline layer resembles a slightly oxygen-rich, oxygenterminated $\mathrm{TiO}_{1.1}(111)$ double layer. It is a polar film with a self-limited thickness. No change of the electronic or geometric structure of the $\mathrm{TiO}_{2}$ substrate surrounding the clusters was observed. These results may help to identify the reactive site responsible for catalytic enhancement in the SMSI effect. They also show the capability of STM to obtain detailed surface investigations of nanosized clusters in special cases.

This work was supported by NSF-CAREER and DoEEPSCoR. The authors thank Dwight R. Jennison for useful discussions.

*Corresponding author.

Email address: diebold@mailhost.tcs.tulane.edu

[1] C. R. Henry, Surf. Sci. Rep. 31, 231 (1998).

[2] S. J. Tauster, S. C. Fung, and R. L. Garten, J. Am. Chem. Soc. 100, 170 (1978).

[3] G. L. Haller and D. E. Resasco, Adv. Catal. 36, 173 (1989).

[4] A. K. Datye, D. S. Kalakkad, and M. H. Yao, J. Catal. 155, 148 (1995).

[5] G. E. Poirer, B. K. Hance, and J. M. White, J. Phys. Chem. 97, 5965 (1998).

[6] A. Berko, G. Menesi, and F. Solymosi, Surf. Sci. 372, 202 (1997).

[7] C. Xu, X. Lai, G. W. Zajac, and D. W. Goodman, Phys. Rev. B 56, 13464 (1997).

[8] A. Berko and F. Solymosi, Surf. Sci. 411, L900 (1998).

[9] P. L. J. Gunter, J. W. Niemandsverdriet, and G. A. Somorjai, Catal. Rev. Sci. Eng. 39, 77 (1997).

[10] A. B. Boffa et al., Surf. Sci. 326, 80 (1995).

[11] F. Pesty, H.-P. Steinrück, and T. E. Madey, Surf. Sci. 339, 83 (1995).

[12] R. A. Bennett, P. Stone, and M. Bowker, Faraday Discuss. Chem. Soc. 114, 267 (1999).

[13] R. T. Baker, E. B. Prestidge, and R. L. Garten, J. Catal. 56, 390 (1979).

[14] M.-H. Yao, in Proceedings of the 51st Annual Meeting of the Microscopy Society of America, edited by G. W. Bailey and C.L. Rieder (MSA, San Francisco, 1993), p. 734.

[15] L. Wang et al., Proc. Int. Congr. Catal. 9th 3, 1253 (1988).

[16] U. Diebold, J.F. Anderson, K.-O. Ng, and D. Vanderbilt, Phys. Rev. Lett. 77, 1322 (1996). 